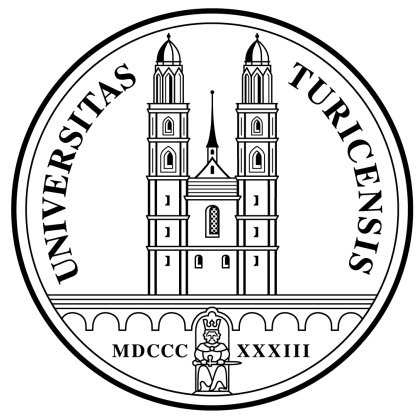

Institute for Empirical Research in Economics

University of Zurich

Working Paper Series

ISSN 1424-0459

Working Paper No. 65

Random Fixed Points

in a Stochastic Solow Growth Model

Klaus Reiner Schenk-Hoppé and Björn Schmalfuss

November 2000 


\title{
Random Fixed Points in a Stochastic Solow Growth Model
}

\author{
Klaus Reiner Schenk-Hoppé ${ }^{1}$ \\ Institute for Empirical Research in Economics, University of Zurich, \\ Blümlisalpstrasse 10,8006 Zürich, Switzerland,klaus@iew.unizh.ch
}

Björn Schmalfuß

Department of Applied Sciences, University of Technology and Applied Sciences, Geusaer Straße,06217 Merseburg, Germany, schmalfuss@in.fh-merseburg.de

\begin{abstract}
This paper presents a complete analysis of a stochastic version of the Solow growth model in which all parameters are ergodic random variables. Applying random dynamical systems theory, we prove that the dynamics and, in particular, the long-run behavior is uniquely determined by a globally attracting stable random fixed point. We also discuss the relation of our approach to that of ergodic Markov equilibria.

Keywords: Solow growth model; random dynamical systems; random fixed points; ergodic Markov equilibria.

JEL classification: C62, E13, O41
\end{abstract}

\section{Introduction}

In the economic literature, ergodic Markov equilibria are commonly used to describe the long-run behavior of stochastic systems. A Markov equilibrium is a probability measure on the state space of some Markov process which is invariant under the transition probability. This notion generalizes the concept of a fixed point of a deterministic dynamical system, see Futia (1982) for a detailed treatment of this approach and Duffie et al. (1994) for a recent account. An analysis of the Solow growth model from this point of view is due to Mirman $(1972,1973)$. He considered the case of independent and identically distributed (i.i.d.) production shocks, possessing a density with respect

\footnotetext{
${ }^{1}$ Research supported in part by the Deutsche Forschungsgemeinschaft under contract Bo 635/8-2 while KRSH was at the Department of Economics, University of Bielefeld, Germany.
} 
to Lebesgue measure (Mirman, 1973), and a case in which both population growth rate and production shocks are uniformly bounded acyclic ergodic Markov processes (Mirman, 1972).

Beside the fact that the concept of an (ergodic) Markov equilibrium makes sense only for systems which are Markov processes, it has the major shortcoming of not being related to sample path stability in general. That is, an ergodic Markov equilibrium does not necessarily provide any information whether sample paths starting at different states converge or diverge for a given sequence of random shocks, i.e. the qualitative long-run behavior of each sample path of an economy is completely described by an ergodic Markov equilibrium from a statistical point of view, but the quantitative behavior of single sample paths as well as the qualitative behavior of ensembles of sample paths is not described by the Markov equilibrium in general.

Our goal in this paper is to introduce and illustrate an equilibrium concept for nonlinear stochastic systems which is related to the dynamical behavior and, in particular, to the sample path stability of such systems. The emphasis here is on the study of the simultaneous motion of several sample paths instead of single sample paths. The concept of a random fixed point provides an alternative stochastic analogue of a deterministic fixed point. In this paper, we apply this concept to derive a complete analysis of a stochastic version of the Solow growth model, Solow (1956) and Swan (1956), in which all parameters are ergodic random variables. We prove that the dynamics (and therefore the long-run behavior) of this stochastic system is uniquely described by a globally attracting stable random fixed point. In particular, sample paths with different initial states behave identical in the long-run (from a statistical as well as from a dynamical point of view) and their stability properties are determined by the random fixed point.

Our study is placed within the framework of random dynamical systems, Arnold (1998). This theory offers a description of stochastic systems from a dynamical systems point of view, and it is more general than a description by stochastic processes. In particular, the theory of random dynamical systems covers Markov processes and hence Markov equilibria. The main tool for our analysis is a random version of the Banach fixed point theorem which is due to Schmalfuß $(1996,1998)$. A version for random difference equations is presented here. We also analyze the relation of random fixed points and Markov equilibria. In a recent paper, Becker and Zilcha (1997) prove existence of random fixed points in a stochastic Ramsey model. However, they cannot carry out a stability analysis of the random fixed point because of the lack of sufficient information about the optimal policy function.

The paper is organized as follows. Section 2 introduces random dynamical systems generated by random difference equations and random fixed points. Section 3 analyzes the stochastic Solow growth model. Two examples are studied in Section 4. The relationship between Markov equilibria and random fixed points is analyzed in Section 5. 


\section{Random dynamical systems with discrete time}

We give a brief introduction to the framework used to study perturbed dynamical systems that are described by random difference equations. The reader is referred to Arnold (1998) for the general theory and for additional information.

Let $(\Omega, \mathcal{F}, \mathbb{P})$ denote a probability space, and let $\theta: \Omega \rightarrow \Omega$ be a measurable invertible mapping with measurable inverse $\theta^{-1}$. We will assume that $\theta$ is ergodic, i.e. $\mathbb{P}$ is ergodic with respect to $\theta$. This mapping is defined with twosided time $\mathbb{Z}$ without loss of generality. The collection $\left(\Omega, \mathcal{F}, \mathbb{P},\left\{\theta^{t}\right\}_{t \in \mathbb{Z}}\right)$ is called an ergodic dynamical system, denoted by $\theta$ for short.

Consider the random difference equation $x_{t+1}=h\left(\theta^{t} \omega, x_{t}\right)$ where the map $h(\omega, \cdot)=: h(\omega): \mathbb{R}^{d} \rightarrow \mathbb{R}^{d}$ is measurable and measurably invertible. Define,

$$
\varphi(t, \omega, x)= \begin{cases}h\left(\theta^{t-1} \omega\right) \circ \ldots \circ h(\omega) x & \text { for } t \geq 1 \\ x & \text { for } t=0 \\ h\left(\theta^{t} \omega\right)^{-1} \circ \ldots \circ h\left(\theta^{-1} \omega\right)^{-1} x & \text { for } t \leq-1\end{cases}
$$

$\varphi(t, \omega, x)$ is the state of a stochastic system (generated by a random map $h$ ) at time $t$ which has been started at $x_{0}=x$ under the perturbation determined by $\omega$. In particular, $\varphi(1, \omega) \equiv h(\omega)$. $h$ is called the generator of $\varphi$.

If $h(\omega): \mathbb{R}^{d} \rightarrow \mathbb{R}^{d}$ is measurable but not invertible, then only the first and the second equation in (1) hold. In particular, the sample path $\varphi(t, \omega, x)$ is only defined for $t \geq 0$.

The family of maps $\varphi(t, \omega, x)$ is called a random dynamical system. That is, $\varphi: \mathbb{T} \times \Omega \times \mathbb{R}^{d} \rightarrow \mathbb{R}^{d},(t, \omega, x) \mapsto \varphi(t, \omega, x)$ is a measurable mapping such that $\varphi(0, \omega)=\operatorname{id}_{\mathbb{R}^{d}}$ and $\varphi(s+t, \omega)=\varphi\left(t, \theta^{s} \omega\right) \circ \varphi(s, \omega)$ for all $s, t \in \mathbb{T}$, and $\omega \in \Omega$. These properties replace the flow property of a deterministic dynamical system that is generated by the iteration of a map.

If the generator $h$ is invertible (non-invertible), then $\mathbb{T}=\mathbb{Z}(\mathbb{T}=\mathbb{N})$. Obviously, $\varphi(t, \omega)$ inherits the regularities (such as continuity or smoothness) of $h$ for $t \geq 0$ and of $h^{-1}$ for $t \leq 0$. Note that the ergodic dynamical system modeling the noise process is defined for time $\mathbb{T}=\mathbb{Z}$ in both invertible and non-invertible case.

Definition $1 A$ random fixed point of a random dynamical system $\varphi$ is a random variable $x^{\star}: \Omega \rightarrow \mathbb{R}^{d}$ such that almost surely

$$
x^{\star}(\theta \omega)=\varphi\left(1, \omega, x^{\star}(\omega)\right):=h\left(\omega, x^{\star}(\omega)\right) .
$$

In the context of random dynamical systems, we will use the notion almost surely (abbreviated a.s.) in the following (non-standard) sense. A statement holds a.s. if there exists a $\theta$-invariant set $\Omega^{\prime} \subset \Omega\left(\theta \Omega^{\prime}=\Omega^{\prime}\right)$ of full $\mathbb{P}$-measure $\left(\mathbb{P}\left(\Omega^{\prime}\right)=1\right)$ such that this statement holds true for all $\omega \in \Omega^{\prime}$. For instance, 
the statement of the ergodic theorem can be understood in this sense, see (Arnold, 1998, App. A.1).

Equation (2) implies $x^{\star}\left(\theta^{t+1} \omega\right)=h\left(\theta^{t} \omega, x^{\star}\left(\theta^{t} \omega\right)\right)=\varphi\left(t+1, \omega, x^{\star}(\omega)\right)$ for all $t$. Hence a random fixed point is a stationary process which satisfies the random difference equation $x_{t+1}(\omega)=h\left(\theta^{t} \omega, x_{t}(\omega)\right)$. Figure 1 depicts the sample path of a random fixed point.

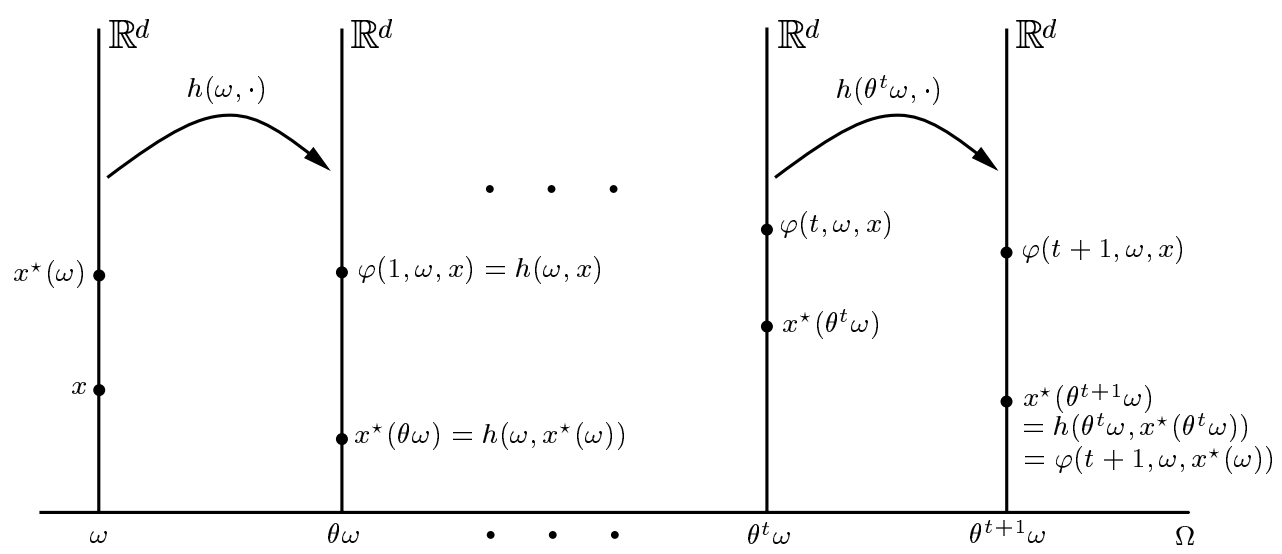

Fig. 1. Random fixed point $x^{\star}$ of a random dynamical system $\varphi$ over the ergodic dynamical system $\theta . \varphi$ is generated by the mapping $h$.

Suppose the perturbation is trivial, i.e. $h(\omega, x) \equiv h(x)$, then the above definition coincides with the notion of a deterministic steady state $x^{\star}=h\left(x^{\star}\right)$.

By ergodicity of $\theta$, the process $x^{\star}\left(\theta^{t} \omega\right)$ is ergodic, and thus if $\mathbb{E}\left|x^{\star}\right|<\infty$ then $\lim _{t \rightarrow \infty} 1 / t \sum_{s=0}^{t} f\left(x^{\star}\left(\theta^{s} \omega\right)\right)=\int f d\left(x^{\star} \mathbb{P}\right)$ almost surely for any integrable $f$. That is the empirical law of the orbit $x^{\star}\left(\theta^{t} \omega\right)$ is well-defined and equal to $x^{\star} \mathbb{P}$. The graph of $x^{\star}$ is an invariant subset of the space $\Omega \times \mathbb{R}^{d}$ with respect to the mapping $(\omega, x) \mapsto(\theta \omega, h(\omega, x))$. Determining a random fixed point is equivalent to solving a (typically infinite) number of coupled equations. The coupling enters through the operator $\theta$ appearing on the left-hand side of (2).

\section{Analysis of a stochastic Solow model}

\subsection{The model}

The stochastic Solow model considered in this paper is given by

$$
k_{t+1}=\frac{\left(1-\delta\left(\theta^{t} \omega\right)\right) k_{t}+\xi\left(\theta^{t} \omega\right) f\left(k_{t}\right)}{1+n\left(\theta^{t} \omega\right)} \equiv h\left(\theta^{t} \omega, k_{t}\right)
$$

$k_{t}$ is the capital per worker in period $t$. (3) is a non-linear random difference equation, describing the stochastic evolution of the capital intensity $k_{t}$ in time. $f: \mathbb{R}_{+} \rightarrow \mathbb{R}_{+}$is a neoclassical production function. $\delta\left(\theta^{t} \omega\right), \xi\left(\theta^{t} \omega\right)$, and $n\left(\theta^{t} \omega\right)$ are ergodic processes that model stationary fluctuations of the rate of depreciation, invested share of output, and population growth rate. The invested 
output share $\xi\left(\theta^{t} \omega\right) f\left(k_{t}\right)$ represents a random saving rate and a multiplicative perturbation of the technology. The case $f(0)=0$ corresponds to an economy in which production of goods is impossible without capital, and in the case $f(0)>0$ goods can be produced with labor as the only input. Zero is a steady state for any sequence of random shocks if $f(0)=0$. For $\delta(\omega) \equiv \delta, \xi(\omega) \equiv s$, and $n(\omega) \equiv n$, we reobtain the deterministic Solow model, Solow (1956).

Binder and Pesaran (1999) show that a similar stochastic Solow model with stochastic population growth and production shocks can be fitted with actual data. Mirman $(1972,1973)$ has studied the existence of Markov equilibria in this and similar models in which the population growth and the production are uniformly bounded acyclic ergodic Markovian processes (Mirman, 1972) and in which the production is subject to i.i.d. shocks possessing a density with respect to Lebesgue measure (Mirman, 1973). For a motivation of a stochastic depreciation rate see Ambler and Paquet (1994, p. 104).

Let us review the results for the deterministic Solow model.

Proposition 2 Assume that $f$ is positive, increasing, strictly concave, and continuously differentiable on $\mathbb{R}_{++}$.

If $\delta+n>0, s>0$, and $f$ satisfies the Inada condition

(i) $0 \leq \lim _{k \rightarrow \infty} f^{\prime}(k)<(\delta+n) / s<\lim _{k \rightarrow 0} f^{\prime}(k) \leq \infty$,

then the deterministic Solow model (i.e. (3) with $\delta(\omega) \equiv \delta, \xi(\omega) \equiv$ s, and $n(\omega) \equiv n)$ possesses a unique non-trivial fixed point $\bar{k}(\delta, n, s)$. The fixed point is stable and globally attracting on $\mathbb{R}_{++}$.

If $f(0)>0$, then no condition on $\lim _{k \rightarrow 0} f^{\prime}(k)$ is needed and $\bar{k}(\delta, n, s)$ is even globally attracting on $\mathbb{R}_{+}$.

Proof. Any non-trivial steady state $\bar{k}$ satisfies $f(\bar{k}) / \bar{k}=(\delta+n) / s$ which is immediate from $(3)$. Since $h(k)=[(1-\delta) k+s f(k)] /[1+n]$ is increasing and strictly concave, there is at most one non-trivial steady state. The Inada condition is equivalent to $0 \leq \lim _{k \rightarrow \infty} f(k) / k<(\delta+n) / s<\lim _{k \rightarrow 0} f(k) / k \leq$ $\infty$ if $f(0)=0$. (For the first condition apply de l'Hôpital's rule.) If $f(0)>0$, then $\lim _{k \rightarrow 0} f(k) / k=\infty$. Hence (i) ensures existence of $\bar{k}:=\bar{k}(\delta, n, s)$, because $f$ is continuous. Since $h$ is strictly concave and $h(k)>k$ for small $k>0$, the derivative $h^{\prime}(\bar{k})<1$ and thus $\bar{k}$ is stable. The global attraction property follows from the facts that $\bar{k}$ is the unique steady state on $\mathbb{R}_{++}, h(k)>k$ for all $0<k<\bar{k}$, and $h(k)<k$ for all $k>\bar{k}$.

\subsection{Main result}

We prove that the dynamics of the stochastic Solow model is determined by a unique stable and globally attracting random fixed point. In particular, it is ensured that the long-run behavior of all sample paths of capital intensities is identical and is determined by the path of this random fixed point. The random fixed point is ergodic and thus the time average of the capital intensity is constant (and equal to the mean value of capital per worker at the 
random fixed point) for all initial states and almost all sample paths of the perturbation.

Theorem 3 Assume the stochastic processes representing the rates of depreciation and population growth, and the product of saving rate and production shocks, respectively, take values $\delta(\omega) \in\left[\delta_{\min }, \delta_{\max }\right] \subset[0,1], n(\omega) \in$ $\left.\left[n_{\min }, n_{\max }\right] \subset\right]-1, \infty\left[\right.$, and $\xi(\omega) \in\left[\xi_{\text {min }}, \infty[\subset] 0, \infty[\right.$ with $\mathbb{E} \xi<\infty$. Assume further that $f$ is non-negative, increasing, strictly concave, and continuously differentiable.

Suppose that

(i) $\delta_{\max }+n_{\max }>0$;

(ii) $0 \leq \lim _{k \rightarrow \infty} f^{\prime}(k)<\frac{\delta_{\max }+n_{\max }}{\xi_{\min }}<\lim _{k \rightarrow 0} f^{\prime}(k) \leq \infty$; and

(iii) $\mathbb{E} \log \frac{1-\delta(\omega)+\xi(\omega) f^{\prime}(\bar{k})}{1+n(\omega)}<0$, where $\bar{k}:=\bar{k}\left(\delta_{\max }, n_{\max }, \xi_{\min }\right)$ is the nontrivial steady state of the deterministic Solow model with respective parameters. $\bar{k}$ is well-defined by conditions (i) and (ii), see Proposition 2.

Then there exists a unique non-trivial random fixed point $k^{\star}$ for the random dynamical system $\varphi$ generated by the stochastic Solow growth model (3). $k^{\star}$ is stable, measurable with respect to the past, and globally attracting on $\mathbb{R}_{++}$, i.e. for all $k>0,\left\|\varphi(t, \omega, k)-k^{\star}\left(\theta^{t} \omega\right)\right\| \rightarrow 0$ as $t \rightarrow \infty$ a.s. Therefore, the long-run behavior of all sample paths is uniquely determined by the random fixed point $k^{\star}$.

If $f(0)>0$, then no condition on $\lim _{k \rightarrow 0} f^{\prime}(k)$ is needed and $k^{\star}$ is even globally attracting on $\mathbb{R}_{+}$.

The random variables $\delta(\omega), n(\omega)$, and $\xi(\omega)$ are arbitrary ergodic processes. In particular, they may be jointly independent as well as correlated processes.

Theorem 3 covers the results on the deterministic Solow model stated in Proposition 2. To see this, it suffices to check that condition (iii) of Theorem 3 is no additional restriction, if all quantities are deterministic. In this case, (iii) becomes $f^{\prime}(\bar{k})<(\delta+n) / \xi$. Since $f(\bar{k}) / \bar{k}=(\delta+n) / \xi$ for each non-trivial steady state (see proof of Proposition 2), it remains to show that $f^{\prime}(\bar{k})<f(\bar{k}) / \bar{k}$. But this follows from strict concavity of $f$.

\subsection{A random fixed point theorem}

This section states an existence theorem for random fixed points used in the proof of the main result. The theorem is a version of a Banach fixed point theorem for nonlinear stochastic systems due to Schmalfuß $(1996,1998)$.

Let $G(\omega) \subset \mathbb{R}^{d}, \omega \in \Omega$, be a random set, i.e. $G(\omega)$ is closed a.s. and $\{\omega \mid G(\omega) \cap U=\emptyset\}$ is measurable for all open sets $U$. In the following we will consider random variables $g(\omega)$ with values in $G(\omega)$.

Suppose $\theta$ is an ergodic dynamical system. Then all paths $g\left(\theta^{t} \omega\right), t \in \mathbb{Z}$, of a random variable $g: \Omega \rightarrow \mathbb{R}^{d}$ grow either super-exponentially fast (i.e. 
$\limsup \operatorname{sum}_{t \rightarrow \infty} \log ^{+}\left\|g\left(\theta^{t} \omega\right)\right\| / t=\infty$ a.s.) or they grow sub-exponentially fast (i.e. $\limsup \sup _{t \rightarrow \infty} \log ^{+}\left\|g\left(\theta^{t} \omega\right)\right\| / t=0$ a.s.), see O'Brien (1982). In the latter case, $g$ is called tempered, and we define

$$
\mathcal{G}:=\{\text { all tempered random variables } g \text { with } g(\omega) \in G(\omega)\}
$$

Temperedness of $g$ is equivalent to

$$
\lim _{t \rightarrow \infty} e^{-\delta t}\left\|g\left(\theta^{t} \omega\right)\right\|=0 \text { for all } \delta>0
$$

Let $\xi\left(\theta^{t} \omega\right)$ be an i.i.d. process, then $\xi$ is tempered if and only if $\mathbb{E}\|\xi\|<\infty$, see Arnold (1998, Prop. 4.1.3). In general, temperedness is a weaker condition than integrability.

Theorem 4 Let $\varphi$ be a random dynamical system such that the map $x \mapsto$ $\varphi(1, \omega, x)$ is continuously differentiable a.s., and let $\theta$ be ergodic. Suppose there exists a random set $G(\omega), \omega \in \Omega$, such that $\mathcal{G}$ is non-empty and

(i) $\varphi\left(1, \theta^{-1} \omega, g\left(\theta^{-1} \omega\right)\right) \in \mathcal{G}$ for all $g \in \mathcal{G}$;

(ii) $\sup _{x \in G(\omega)} \log \left\|\frac{\partial \varphi(1, \omega, x)}{\partial x}\right\| \leq c(\omega)$ with $\mathbb{E} c(\omega)<0$; and

(iii) if, for some $g \in \mathcal{G}, \varphi\left(t, \theta^{-t} \omega, g\left(\theta^{-t} \omega\right)\right)$ is a Cauchy sequence for all $\omega \in \Omega$, then its limit is in $\mathcal{G}$.

Then there exists a random variable $g^{\star} \in \mathcal{G}$ such that a.s.

(a) $\varphi\left(1, \omega, g^{\star}(\omega)\right)=g^{\star}(\theta \omega)$;

(b) $\lim _{t \rightarrow \infty}\left\|\varphi(t, \omega, g(\omega))-g^{\star}\left(\theta^{t} \omega\right)\right\|=0$ for all $g \in \mathcal{G}$; and

(c) $g^{\star}(\omega)$ is uniquely determined.

Conditions (i) - (iii) are invariance, uniform average contractivity, and completeness assumptions, respectively. The assertion is the existence of a random fixed point $g^{\star}$ with $\mathcal{G}$ being a subset of its basin of attraction. For trivial noise, the theorem is the Banach fixed point theorem for differentiable mappings.

The long-run behavior of all sample paths with initial values $g(\omega) \in G(\omega)$ is completely specified by the orbit $g^{\star}\left(\theta^{t} \omega\right)$. In particular the (space) average $\lim _{t \rightarrow \infty} 1 / t \sum_{s=0}^{t} \varphi(s, \omega, g(\omega))=\lim _{t \rightarrow \infty} 1 / t \sum_{s=0}^{t} g^{\star}\left(\theta^{s} \omega\right)=\mathbb{E} g^{\star}$ and thus is constant a.s., if $g^{\star}$ is integrable.

Suppose $g^{\star}(\omega)>0$. Then the growth rate $\gamma_{g^{\star}}(\omega):=\left(g^{\star}(\theta \omega)-g^{\star}(\omega)\right) / g^{\star}(\omega)$ is tempered, by temperedness of $g^{\star}$, and $\mathbb{E} \log \left(1+\gamma_{g^{\star}}(\omega)\right)=0$, if $\log g^{\star}$ is integrable.

Proposition 5 If the set of tempered random variables $\mathcal{G}$ in Theorem 4 contains a constant random variable $g(\omega) \equiv g$, then the random fixed point $g^{\star}: \Omega \rightarrow \mathbb{R}^{d}$ is measurable with respect to the past $\mathcal{F}^{-}:=\sigma\left\{\omega \mapsto \varphi\left(s, \theta^{-t} \omega\right) \mid\right.$ $0 \leq s \leq t\}$.

Proof. The conclusion $\lim _{t \rightarrow \infty}\left\|\varphi(t, \omega, g(\omega))-g^{\star}\left(\theta^{t} \omega\right)\right\|=0$ for all $g \in \mathcal{G}$ in Theorem 4 implies (by stationarity of $\theta$ )

$$
\left\|\varphi\left(t, \theta^{-t} \omega, g\left(\theta^{-t} \omega\right)\right)-g^{\star}(\omega)\right\| \rightarrow 0 \quad \text { as } t \rightarrow \infty \text { in probability. }
$$


If $g(\omega) \equiv g$ is an element of $\mathcal{G}$, then $\varphi\left(t, \theta^{-t} \omega, g\right)$ converges as $t \rightarrow \infty$ and its limit is equal to $g^{\star}(\omega)$ (a.s.). Thus $g^{\star}(\omega)$ is measurable with respect to the $\sigma$-algebra $\sigma\left\{\omega \mapsto \varphi\left(t, \theta^{-t} \omega\right) \mid 0 \leq t\right\} \subset \mathcal{F}^{-}$.

\subsection{Proof of the main result}

Proof of Theorem 3 This proof is an application of the random fixed point theorem 4. We first define a set $G$ and then show that the conditions of Theorem 4 are satisfied. The global attraction property and $\mathcal{F}^{-}$-measurability is proved at the end of this proof.

(a) Definition of $G$. Let $G:=\left[\bar{k}\left(\delta_{\max }, n_{\max }, \xi_{\min }\right), \infty[. G(\omega) \equiv G\right.$ is a random set by closedness of $G$. $\mathcal{G}$ is non-empty because it contains all constant functions with values larger than $\bar{k}$. Since $\bar{k}:=\bar{k}\left(\delta_{\max }, n_{\max }, \xi_{\min }\right)>0$, the application of Theorem 4 will yield a non-trivial random fixed point $k^{\star}$.

(b) We check condition (i) of Theorem 4: invariance of $\mathcal{G}$.

It is a simple exercise to check that $G$ is forward invariant (i.e. if $k \in$ $G$, then $h(\omega, k) \in G$ for all $\omega)$, because $h(\omega, k) \geq \bar{k}$ for all $k \in G, \omega \in$ $\Omega$. (Observe that the function $h$ decreases as $\delta(\omega)$ or $n(\omega)$ increase, or $\xi(\omega)$ decreases. $\bar{k}\left(\delta_{\max }, n_{\max }, \xi_{\min }\right)$ is hence the smallest non-trivial fixed point of all deterministic maps $h(k)$ associated to (3) with parameters $\delta \in\left[\delta_{\min }, \delta_{\max }\right]$, $n \in\left[n_{\min }, n_{\max }\right]$, and $\xi \in\left[\xi_{\text {min }}, \infty[\right.$.$) Therefore, \varphi\left(1, \theta^{-1} \omega, g\left(\theta^{-1} \omega\right)\right) \in G(\omega) \equiv$ $G$ for all $g \in \mathcal{G}$.

We next show that $\omega \mapsto \varphi\left(1, \theta^{-1} \omega, g\left(\theta^{-1} \omega\right)\right)$ is tempered for all $g \in \mathcal{G}$. This yields condition (i) of Theorem 4 .

Concavity of $f$ implies $f(k) \leq f(y)+f^{\prime}(y) k$ for all $k$ with an arbitrarily fixed $y>0$. (Recall that $f^{\prime}(0)=\infty$ is not excluded in the theorem.) Therefore,

$$
\varphi(1, \omega, k) \leq \frac{1-\delta(\omega)+\xi(\omega) f^{\prime}(y)}{1+n(\omega)} k+\frac{\xi(\omega) f(y)}{1+n_{\min }}
$$

Since $\xi$ is tempered (which is implied by $\mathbb{E} \xi<\infty$ (Arnold, 1998, Prop. 4.1.3)), $1 /(1+n(\omega)) \leq 1 /\left(1+n_{\text {min }}\right)$, and sums as well as products of finitely many tempered random variables are tempered, the above estimate ensures temperedness of $\varphi\left(1, \theta^{-1} \omega, g\left(\theta^{-1} \omega\right)\right)$.

(c) We check condition (ii) of Theorem 4: contraction. This follows directly from assumption (iii), because $f^{\prime}$ is decreasing and thus assumes its maximum at the minimal element of $G$, i.e. $\sup _{k \in G} f^{\prime}(k)=f^{\prime}(\bar{k})$.

(d) We check condition (iii) of Theorem 4: convergence. Let $g \in \mathcal{G}$, and suppose that $\varphi\left(t, \theta^{-t} \omega, g\left(\theta^{-t} \omega\right)\right)$ is a Cauchy sequence for all $\omega$. Then the limit is in $G(\omega)$ for all $\omega$, because $G(\omega) \equiv G$ is forward invariant and complete. To ensure (iii) it thus suffices to check that $\lim _{t \rightarrow \infty} \varphi\left(t, \theta^{-t} \omega, g\left(\theta^{-t} \omega\right)\right.$ ) (assumed to exists) is a tempered random variable.

We need the following observation to ensure the temperedness. Define the affine random difference equation

$$
x_{t+1}=a\left(\theta^{t} \omega\right) x_{t}+b\left(\theta^{t} \omega\right),
$$


with

$$
a(\omega):=\frac{1-\delta(\omega)+\xi(\omega) f^{\prime}(\bar{k})}{1+n(\omega)} \quad \text { and } \quad b(\omega):=\frac{\xi(\omega) f(\bar{k})}{1+n_{\min }}
$$

Then $\lim _{t \rightarrow \infty} \varphi\left(t, \theta^{-t} \omega, g\left(\theta^{-t} \omega\right)\right)$ is bounded from above by the random fixed point, say $x^{\star}(\omega)$, of (6). This claim follows from (5) and the fact that $x^{\star}(\omega)$ is stable by assumption (iii) and hence attracts any tempered random variable. We next prove existence and temperedness of $x^{\star}(\omega)$.

Existence of $x^{\star}(\omega)$ : The only candidate for a random fixed point is

$$
x^{\star}(\omega):=b\left(\theta^{-1} \omega\right)+\sum_{i=1}^{\infty} b\left(\theta^{-(i+1)} \omega\right) \prod_{j=1}^{i} a\left(\theta^{-j} \omega\right)
$$

see Arnold and Crauel (1992). $x^{\star}(\omega)$ is well defined because $\mathbb{E} \log a<0$ and $\mathbb{E} b<\infty$ : Observe that for any $0<\varepsilon<-\mathbb{E} \log a$ there exists a $t(\varepsilon, \omega)$ such that $\prod_{j=1}^{i} a\left(\theta^{-j} \omega\right) \leq e^{-\varepsilon i}$ for all $i \geq t(\varepsilon, \omega)$. Now, temperedness of $b(\omega)$, which is implied by $\mathbb{E} b<\infty$ (Arnold, 1998, Prop. 4.1.3), gives the a.s. existence.

Temperedness of $x^{\star}(\omega)$ : Recall that $\mathbb{E} \log a<0$ and $b$ is tempered. Therefore, for all $\varepsilon>0$ there exists an $s_{0}(\omega, \varepsilon)$ such that for all $s \geq s_{0}(\omega, \varepsilon)$, $\log b\left(\theta^{-s} \omega\right) \leq \varepsilon s$ and $\left|\sum_{j=1}^{s}\left(\log a\left(\theta^{-j} \omega\right)-\mathbb{E} \log a\right)\right| \leq \varepsilon s$.

Since $a(\omega)>0$ and $b(\omega)>0$, one has the following representation

$$
\begin{aligned}
x^{\star}\left(\theta^{-t} \omega\right)= & \exp \log b\left(\theta^{-(t+1)} \omega\right) \\
& +\sum_{i=1}^{\infty} \exp \left[\log b\left(\theta^{-(i+1+t)} \omega\right)+\sum_{j=1}^{t+i}\left(\log a\left(\theta^{-j} \omega\right)-\mathbb{E} \log a\right)\right. \\
& \left.\quad-\sum_{j=1}^{t}\left(\log a\left(\theta^{-j} \omega\right)-\mathbb{E} \log a\right)+i \mathbb{E} \log a\right]
\end{aligned}
$$

Thus, for $\varepsilon<|\mathbb{E} \log a| / 2$ and all $t \geq s_{0}(\omega, \varepsilon)$ it is

$$
\begin{aligned}
x^{\star}\left(\theta^{-t} \omega\right) & \leq \exp (\varepsilon(t+1))+\sum_{i=1}^{\infty} \exp (\varepsilon((i+1+t)+(t+i)+t)+i \mathbb{E} \log a) \\
& \leq \exp (\varepsilon(t+1))+\exp (\varepsilon(3 t+1)) \frac{\exp (2 \varepsilon+\mathbb{E} \log a)}{1-\exp (2 \varepsilon+\mathbb{E} \log a)}
\end{aligned}
$$

Temperedness of $x^{\star}(\omega)$ can now be seen using (4). Fix any $\delta>0$, then for each $\varepsilon$ such that $0<\varepsilon<\max \{\delta / 3,-(\mathbb{E} \log a) / 2\}$ one has $e^{-\delta t} x^{\star}\left(\theta^{-t} \omega\right) \rightarrow 0$.

We thus have proved (d) and therefore the application of the random fixed point theorem yields all assertions claimed except the global attraction property.

(e) Global attraction property. If all noise processes are trivial, then $\bar{k}(\delta, n, \xi)$ is a steady state that is globally attracting on $\mathbb{R}_{++}$, see Proposition 2 and the remark given after Theorem 3. Now, suppose that at least one noise process is non-trivial. Assume that this is true for the rate of depreciation, i.e. there 
exists a $\delta$ such that $\delta_{\min }<\delta<\delta_{\max }$ and $0<\mathbb{P}\{\delta(\omega) \leq \delta\}<1$. All other cases can be treated completely analogous.

Observe first that $\varphi(1, \omega, k) \equiv h(\omega, k) \geq k$ for all $\omega \in \Omega$ and all $k \leq \bar{k}:=$ $\bar{k}\left(\delta_{\max }, n_{\text {max }}, \xi_{\text {min }}\right)$. Further, for each $k>0$ there exists a $t(\omega, k)$ such that, if $\delta\left(\theta^{s} \omega\right) \leq \delta$ for all $0 \leq s \leq t(\omega, k)$, then $\varphi(t(\omega, x), \omega, k)>\bar{k}$. Property (1) ensures that it suffices to have $\delta\left(\theta^{s} \omega\right) \leq \delta$ for at least $t(\omega, k)$ different times less than some finite $T(\omega, k)$ to get $\varphi(u, \omega, k)>\bar{k}$ for all $u \geq T(\omega, k)$. Thus, if the existence of such a $T(\omega, k)$ is proved we are done.

Ergodicity of $\theta$ implies $\lim _{t \rightarrow \infty} 1 / t \sum_{s=0}^{t} 1_{[0, \delta]} \delta\left(\theta^{s} \omega\right)=\mathbb{P}\{\omega \mid \delta(\omega) \leq \delta\}>0$ and therefore $\mathbb{P}\left\{\omega \mid \delta\left(\theta^{s} \omega\right) \leq \delta\right.$ for infinitely many $\left.s \geq 0\right\}=1$. But this yields existence of a finite $T(\omega, k)$ for all $\omega$ and all $k$ which possesses the property required to complete $(\mathrm{e})$.

(f) Uniqueness and $\mathcal{F}^{-}$-measurability. Uniqueness of the random fixed point on $\mathbb{R}_{++}$follows immediately from the global attraction property. $\mathcal{F}^{-}$-measurability follows from Proposition 5, cf. step (a) of this proof.

\section{Examples and sufficient conditions}

First we derive a simple sufficient condition for the contraction condition (iii) of Theorem 3. This condition can be rewritten as $\mathbb{E} \log (1-\delta(\omega)+$ $\left.\xi(\omega) f^{\prime}(\bar{k})\right)<\mathbb{E} \log (1+n(\omega))$. Using that $\log$ is concave (which implies $\mathbb{E} \log \leq$ $\log \mathbb{E}$ by Jensen's inequality), we obtain the sufficient condition $\mathbb{E}(1-\delta(\omega)+$ $\left.\xi(\omega) f^{\prime}(\bar{k})\right)<\exp (\mathbb{E} \log (1+n(\omega)))$ which is equivalent to

$$
f^{\prime}\left(\bar{k}\left(\delta_{\text {max }}, n_{\text {max }}, \xi_{\text {min }}\right)\right)<\frac{\mathbb{E} \delta(\omega)-1+\exp (\mathbb{E} \log (1+n(\omega)))}{\mathbb{E} \xi(\omega)}
$$

Using that $n(\omega) \geq n_{m i n},(7)$ follows from

$$
f^{\prime}\left(\bar{k}\left(\delta_{\text {max }}, n_{\text {max }}, \xi_{\text {min }}\right)\right)<\frac{\mathbb{E} \delta(\omega)+n_{\text {min }}}{\mathbb{E} \xi(\omega)}
$$

Therefore, (8) implies condition (iii) of Theorem 3.

Example 1. Consider the Cobb-Douglas production function $f(k)=k^{\alpha}$ with $0<\alpha<1$. The Inada condition (ii) of Theorem 3 is satisfied for all $\alpha$. Equation (8) (and thus condition (iii) of Theorem 3) is fulfilled, if

$$
\alpha<\frac{\xi_{\min }}{\mathbb{E} \xi} \frac{\mathbb{E} \delta(\omega)+n_{\min }}{\delta_{\max }+n_{\max }}
$$

It is straightforward to check that $\bar{k}=\left(\left(\delta_{\max }+n_{\max }\right) / \xi_{\min }\right)^{\frac{1}{\alpha-1}}$ and thus $f^{\prime}(\bar{k})=\alpha\left(\delta_{\max }+n_{\max }\right) / \xi_{\min }$. Inserting the last term into (8) immediately yields the above assumption on $\alpha$.

Example 2. Consider the CES production function $f(k)=\left(1-A+A k^{\alpha}\right)^{1 / \alpha}$ with $0 \neq \alpha<1$, and $0<A<1$. Conditions (ii) and (iii) of Theorem 3 are 
satisfied if

$$
A<\min \left\{\left(\frac{\delta_{\max }+n_{\max }}{\xi_{\min }}\right)^{\alpha}, \frac{\xi_{\min }^{1-\alpha}}{\mathbb{E} \xi} \frac{\mathbb{E} \delta+n_{\min }}{\left(\delta_{\max }+n_{\max }\right)^{1-\alpha}}\right\}
$$

Observing that $f(k) / k=\left(A+(1-A) k^{-\alpha}\right)^{1 / \alpha} \rightarrow A^{1 / \alpha}$ as $k \rightarrow \infty$, it is clear that the Inada condition (ii) follows from our first assumption on $A$. Elementary calculations further yield

$$
\bar{k}=\left(\frac{1-A}{\left(\frac{\delta_{\max }+n_{\max }}{\xi_{\min }}\right)^{\alpha}-A}\right)^{1 / \alpha} \text { and hence } f^{\prime}(\bar{k})=A\left(\frac{\delta_{\max }+n_{\max }}{\xi_{\min }}\right)^{1-\alpha}
$$

where $\bar{k}>0$ by the first assumption on $A$. Using (8), we obtain that (iii) is implied by our second condition on $A$.

\section{Markov equilibria and random fixed points}

We recall the definition of a Markov equilibrium which is a stochastic analogue of a deterministic steady state, see e.g. Futia (1982) and Duffie et al. (1994). Consider the random difference equation $x_{t+1}=h\left(\xi\left(\theta^{t} \omega\right), x_{t}\right)$ on $\mathbb{R}^{d}$, where $h: \mathbb{R}^{m} \times \mathbb{R}^{d} \rightarrow \mathbb{R}^{d}$ is a measurable mapping and $\xi\left(\theta^{t} \omega\right)$ is an $\mathbb{R}^{m}$-valued i.i.d. process, i.e. let $\mu$ denotes the distribution of $\xi$, the associated ergodic dynamical system is given by $\left(\left(\mathbb{R}^{m}\right)^{\mathbb{Z}}, \mathcal{B}\left(\mathbb{R}^{m}\right)^{\mathbb{Z}}, \mu^{\mathbb{Z}}\right)$ and $\xi\left(\theta^{t} \omega\right)=\xi(\omega(t+\cdot))=$ $\omega(t)$. The resulting random sequence $x_{t}$ is a homogeneous Markov process with transition probability $P(x, B)=\mathbb{P}\left\{x_{t+1} \in B \mid x_{t}=x\right\}$. A probability measure $\rho$ is called a Markov equilibrium if, for any set $B \in \mathcal{B}\left(\mathbb{R}^{d}\right)$,

$$
\rho(B)=\int_{\mathbb{R}^{d}} P(x, B) \rho(d x) .
$$

Equation (9) implies that the process $x_{t}$ has the distribution $\rho$ for all $t$, if the initial value $x_{0}$ has the distribution $\rho$. This concept carries over to the case of an ergodic Markov process $\xi$.

Arnold (1998, Thm. 2.1.8) yields the following result on the relationship between random fixed points and ergodic Markov equilibria.

Corollary 6 Suppose $x^{\star}(\omega)$ is a random fixed point of the random dynamical system generated by $x_{t+1}=h\left(\xi\left(\theta^{t} \omega\right), x_{t}\right)$ and $\xi\left(\theta^{t} \omega\right)$ is an $\mathbb{R}^{m}$-valued i.i.d. process. Then the probability measure $\rho(B):=\mathbb{P}\left\{x^{\star}(\omega) \in B\right\}=x^{\star} \mathbb{P}(B)$ is an ergodic Markov equilibrium, if $x^{\star}(\omega)$ is measurable with respect to the $\sigma$ algebra $\sigma\left\{\omega \mapsto \xi\left(\theta^{t} \omega\right) \mid t<0\right\}$ (which corresponds to the past of the noise process).

The following result is immediate from Theorem 3, Proposition 5, and Corollary 6. 
Corollary 7 Suppose the assumptions of Theorem 3 are satisfied. If the process $(\delta(\omega), n(\omega), \xi(\omega))$ is i.i.d., then the stochastic Solow model possesses a unique ergodic Markov equilibrium on $\mathbb{R}_{++}$.

This result applies e.g. if the fluctuations of the parameters are caused by economy wide i.i.d. shocks and each parameter is a function of this shock, or if the parameters are pairwise independent i.i.d. processes.

\section{References}

Ambler, S. and A. Paquet, 1994, Stochastic depreciation and the business cycle, International Economic Review 35, 101-116.

Arnold, L., 1998, Random Dynamical Systems (Springer-Verlag, New York).

Arnold, L. and H. Crauel, 1992, Iterated function systems and multiplicative ergodic theory, in: M. Pinsky and V. Wihstutz, eds., Diffusion processes and related problems in analysis, Vol. II: Stochastic flows, Progress in Probability Vol. 27 (Birkhäuser, Basel) 283-305.

Becker, R. and I. Zilcha, 1997, Stationary Ramsey equilibria under uncertainty, Journal of Economic Theory 75, 122-140.

Binder, M. and M. H. Pesaran, 1999, Stochastic growth models and their econometric implications, Journal of Economic Growth 4, 139-183.

Duffie, D., J. Geanakoplos, A. Mas-Colell and A. McLennan, 1994, Stationary Markov equilibria, Econometrica 62, 745-781.

Futia, C. A., 1982, Invariant distributions and the limiting behavior of Markovian economic models, Econometrica 50, 377-408.

Mirman, L. J., 1972, On the existence of steady state measures for one sector growth models with uncertain technology, International Economic Review 13, 271-286.

Mirman, L. J., 1973, The steady state behavior of a class of one sector growth models with uncertain technology, Journal of Economic Theory 6, 219-242.

O'Brien, G. L., 1982, The occurrence of large values in stationary sequences, Probability Theory and Related Fields 61, 347-353.

Schmalfuß, B., 1996, A random fixed point theorem based on Lyapunov exponents, Random \& Computational Dynamics 4, 257-268.

Schmalfuß, B., 1998, A random fixed point theorem and the random graph transformation, Journal of Mathematical Analysis and Applications 225, 91-113.

Solow, R. M., 1956, A contribution to the theory of economic growth, Quarterly Journal of Economics 70, 65-94.

Swan, T. W., 1956, Economic growth and capital accumulation, Economic Record 32, 334-361. 


\section{Working Papers of the Institute for Empirical Research in Economics}

No.

1. Rudolf Winter-Ebmer and Josef Zweimüller: Firm Size Wage Differentials in Switzerland: Evidence from Job Changers, February 1999

2. Bruno S. Frey and Marcel Kucher: History as Reflected in Capital Markets: The Case of World War II, February 1999

3. Josef Falkinger, Ernst Fehr, Simon Gächter and Rudolf Winter-Ebmer: A Simple Mechanism for the Efficient Provision of Public Goods - Experimental Evidence, February 1999

4. Ernst Fehr and Klaus M. Schmidt: A Theory of Fairness, Competition and Cooperation, April 1999

5. Markus Knell: Social Comparisons, Inequality, and Growth, April 1999

6. Armin Falk and Urs Fischbacher: A Theory of Reciprocity, July 2000

7. Bruno S. Frey and Lorenz Goette: Does Pay Motivate Volunteers?, May 1999

8. Rudolf Winter-Ebmer and Josef Zweimüller: Intra-firm Wage Dispersion and Firm Performance, May 1999

9. Josef Zweimüller: Schumpeterian Entrepreneurs Meet Engel's Law: The Impact of Inequality on InnovationDriven Growth, May 1999

10. Ernst Fehr and Simon Gächter: Cooperation and Punishment in Public Goods Experiments, June 1999

11. Rudolf Winter-Ebmer and Josef Zweimüller: Do Immigrants Displace Young Native Workers: The Austrian Experience, June 1999

12. Ernst Fehr and Jean-Robert Tyran: Does Money Illusion Matter?, June 1999

13. Stefan Felder and Reto Schleiniger: Environmental Tax Reform: Efficiency and Political Feasibility, July 1999

14. Bruno S. Frey: Art Fakes - What Fakes?, An Economic View, July 1999

15. Bruno S. Frey and Alois Stutzer: Happiness, Economy and Institutions, July 1999

16. Urs Fischbacher, Simon Gächter and Ernst Fehr: Are People Conditionally Cooperative? Evidence from a Public Goods Experiment, July 2000

17. Armin Falk, Ernst Fehr and Urs Fischbacher: On the Nature of Fair Behavior, August 1999

18. Vital Anderhub, Simon Gächter and Manfred Königstein: Efficient Contracting and Fair Play in a Simple Principal-Agent Experiment, September 2000

19. Simon Gächter and Armin Falk: Reputation or Reciprocity?, September 1999

20. Ernst Fehr and Klaus M. Schmidt: Fairness, Incentives, and Contractual Choices, September 1999

21. Urs Fischbacher: $z$-Tree - Experimenter's Manual, September 1999

22. Bruno S. Frey and Alois Stutzer: Maximising Happiness?, October 1999

23. Alois Stutzer: Demokratieindizes für die Kantone der Schweiz, October 1999

24. Bruno S. Frey: Was bewirkt die Volkswirtschaftslehre?, October 1999

25. Bruno S. Frey, Marcel Kucher and Alois Stutzer: Outcome, Process \& Power in Direct Democracy, November 1999

26. Bruno S. Frey and Reto Jegen: Motivation Crowding Theory: A Survey of Empirical Evidence, November 1999

27. Margit Osterloh and Bruno S. Frey: Motivation, Knowledge Transfer, and Organizational Forms, November 1999

28. Bruno S. Frey and Marcel Kucher: Managerial Power and Compensation, December 1999

29. Reto Schleiniger: Ecological Tax Reform with Exemptions for the Export Sector in a two Sector two Factor Model, December 1999

30. Jens-Ulrich Peter and Klaus Reiner Schenk-Hoppé: Business Cycle Phenomena in Overlapping Generations Economies with Stochastic Production, December 1999

31. Josef Zweimüller: Inequality, Redistribution, and Economic Growth, January 2000

32. Marc Oliver Bettzüge and Thorsten Hens: Financial Innovation, Communication and the Theory of the Firm, January 2000

33. Klaus Reiner Schenk-Hoppé: Is there a Golden Rule for the Stochastic Solow Growth Model? January 2000

34. Ernst Fehr and Simon Gächter: Do Incentive Contracts Crowd out Voluntary Cooperation? February 2000

35. Marc Oliver Bettzüge and Thorsten Hens: An Evolutionary Approach to Financial Innovation, July 2000

36. Bruno S. Frey: Does Economics Have an Effect? Towards an Economics of Economics, February 2000

37. Josef Zweimüller and Rudolf Winter-Ebmer: Firm-Specific Training: Consequences for Job-Mobility, March 2000

The Working Papers of the Institute for Empirical Research in Economics can be downloaded in PDF-format from http://www.unizh.ch/iew/wp/ 


\section{Working Papers of the Institute for Empirical Research in Economics}

No.

38. Martin Brown, Armin Falk and Ernst Fehr: Contract Inforcement and the Evolution of Longrun Relations, March 2000

39. Thorsten Hens, Jörg Laitenberger and Andreas Löffler: On Uniqueness of Equilibria in the CAPM, July 2000

40. Ernst Fehr and Simon Gächter: Fairness and Retaliation: The Economics of Reciprocity, March 2000

41. Rafael Lalive, Jan C. van Ours and Josef Zweimüller: The Impact of Active Labor Market Programs and Benefit Entitlement Rules on the Duration of Unemployment, March 2000

42. Reto Schleiniger: Consumption Taxes and International Competitiveness in a Keynesian World, April 2000

43. Ernst Fehr and Peter K. Zych: Intertemporal Choice under Habit Formation, May 2000

44. Ernst Fehr and Lorenz Goette: Robustness and Real Consequences of Nominal Wage Rigidity, May 2000

45. Ernst Fehr and Jean-Robert Tyran: Does Money Illusion Matter? REVISED VERSION, May 2000

46. Klaus Reiner Schenk-Hoppé: Sample-Path Stability of Non-Stationary Dynamic Economic Systems, Juni 2000

47. Bruno S. Frey: A Utopia? Government without Territorial Monopoly, June 2000

48. Bruno S. Frey: The Rise and Fall of Festivals, June 2000

49. Bruno S. Frey and Reto Jegen: Motivation Crowding Theory: A Survey of Empirical Evidence, REVISED VERSION, June 2000

50. Albrecht Ritschl and Ulrich Woitek: Did Monetary Forces Cause the Great Depression? A Bayesian VAR Analysis for the U.S. Economy, July 2000

51. Alois Stutzer and Rafael Lalive: The Role of Social Work Norms in Job Searching and Subjective Well-Being, July 2000

52. Iris Bohnet, Bruno S. Frey and Steffen Huck: More Order with Less Law: On Contract Enforcement, Trust, and Crowding, July 2000

53. Armin Falk and Markus Knell: Choosing the Joneses: On the Endogeneity of Reference Groups, July 2000

54. Klaus Reiner Schenk-Hoppé: Economic Growth and Business Cycles: A Critical Comment on Detrending Time Series, August 2000

55. Armin Falk, Ernst Fehr and Urs Fischbacher: Appropriating the Commons - A Theoretical Explanation, September 2000

56. Bruno S. Frey and Reiner Eichenberger: A Proposal for a Flexible Europe, August 2000

57. Reiner Eichenberger and Bruno S. Frey: Europe's Eminent Economists: A Quantitative Analysis, September 2000

58. Bruno S. Frey: Why Economists Disregard Economic Methodology, September 2000

59. Armin Falk, Ernst Fehr, Urs Fischbacher: Informal Sanctions, September 2000

60. Rafael Lalive: Did we Overestimate the Value of Health?, October 2000

61. Matthias Benz, Marcel Kucher and Alois Stutzer: Stock Options: the Managers' Blessing. Institutional Restrictions and Executive Compensation, October 2000

62. Simon Gächter and Armin Falk: Work motivation, institutions, and performance, October 2000

63. Armin Falk, Ernst Fehr and Urs Fischbacher: Testing Theories of Fairness - Intentions Matter, September 2000

64. Ernst Fehr and Klaus Schmidt: Endogenous Incomplete Contracts, November 2000

65. Klaus Reiner Schenk-Hoppé and Björn Schmalfuss: Random fixed points in a stochastic Solow growth model, November 2000

66. Leonard J. Mirman and Klaus Reiner Schenk-Hoppé: Financial Markets and Stochastic Growth, November 2000

The Working Papers of the Institute for Empirical Research in Economics can be downloaded in PDF-format from http://www.unizh.ch/iew/wp/

Institute for Empirical Research in Economics, Blümlisalpstr. 10, 8006 Zürich, Switzerland

Phone: 004116343705 Fax: 004116344907 E-mail: bibiewzh@iew.unizh.ch 